\title{
1 Modelling invasive pathogen load from non-destructive sampling data
}

2

3 Natália Martínkováa, ${ }^{\mathrm{a} b^{*}}$, Pavel Škrabánek ${ }^{\mathrm{c}}$, Jiri Pikula ${ }^{\mathrm{d}}$

4

5 a Institute of Vertebrate Biology, Czech Academy of Sciences, Květná 8, 60365 Brno,

6 Czech Republic; email: martinkova@ivb.cz.

$7 \quad{ }^{\mathrm{b}}$ Institute of Biostatistics and Analyses, Masaryk University, Kamenice 3, 62500

8 Brno, Czech Republic.

$9 \quad{ }^{c}$ Institute of Automation and Computer Science, Faculty of Mechanical Engineering,

10 Brno University of Technology, Technická 2896/2, 61669 Brno, Czech Republic;

11 email: pavel.skrabanek@vut.cz.

$12{ }^{\mathrm{d}}$ Department of Ecology and Diseases of Game, Fish and Bees, University of

13 Veterinary and Pharmaceutical Sciences Brno, Palackého třída 1946/1, 61242 Brno,

14 Czech Republic; email: pikulaj@vfu.cz.

15

16 *Corresponding author: martinkova@ivb.cz 


\section{Abstract}

21 Where microbes colonizing skin surface may help maintain organism homeostasis,

22 those that invade living skin layers cause disease. In bats, white-nose syndrome is a

23 fungal skin infection that affects animals during hibernation and may lead to mortality

24 in severe cases. Here, we inferred the amount of fungus that had invaded skin tissue

25 of diseased animals. We used simulations to estimate the unobserved disease severity

26 in a non-lethal wing punch biopsy and to relate the simulated pathology to the

27 measured fungal load in paired biopsies. We found that a single white-nose syndrome

28 skin lesion packed with spores and hyphae of the causative agent, Pseudogymnoascus

29 destructans, contains 48.93 pg of the pathogen DNA, which amounts to about $1560 P$.

30 destructans genomes in one skin lesion. Relating the information to the known UV

31 fluorescence in Nearctic and Palearctic bats shows that Nearctic bats carry about 1.7

$32 \mu \mathrm{g}$ of fungal DNA per $\mathrm{cm}^{2}$, whereas Palearctic bats have $0.04 \mu \mathrm{g} \mathrm{cm}{ }^{-2}$ of $P$.

33 destructans DNA. With the information on the fungal load that had invaded the host

34 skin, the researchers can now calculate disease severity as a function of invasive

35 fungal growth using non-destructive UV light transillumination of each bat $\square \mathrm{s}$ wing

36 membranes. Our results will enable and promote thorough disease severity assessment

37 in protected bat species without the need for extensive animal and laboratory labor

38 sacrifices.

39

40 Keywords: pathogen load; skin lesion; fungal infection; Pseudogymnoascus

41 destructans; white-nose syndrome; bat; UV light diagnostics 


\section{1. Introduction}

45 Association of pathogen load with infections transmission stands at the forefront of 46 epidemiological dynamics models, where high pathogen load increases chances of 47 transmission (Wilson et al., 2008). The amount of the pathogen that can infect a new host represents an infectious dose. In natural condition, the infectious dose needs to be transmitted through pathogen shedding via aerosol, direct or vector-mediated oral or

50 bodily fluids exchange. As such, the pathogen is often quantified in sputum, feces, 51 urine or blood, but such measures do not directly reflect the systemic infection or 52 disease severity.

53 Clinical data support that the overall pathogen load positively correlates with disease 54 severity (Franz et al., 2010; van der Poll and Opal, 2008). Technical and ethical hurdles hinder estimation of the overall load of the pathogen, where the pathogen may occur in different tissues in differing quantities during disease progression (Cunnington, 2015). Thorough sampling can then be possible during autopsy with pathogen quantification in multiple tissue samples, which is sadly late for the patient.

Research in infectious diseases aims to prevent lethal outcomes and non-lethal methods must be preferred.

61 In case of white-nose syndrome (WNS), a fungal infection caused by

62 Pseudogymnoascus destructans in hibernating bats (Blehert et al., 2009; Lorch et al.,

63 2011), the disease severity can be estimated from histopathologic examination of

64 wing membrane tissue (Meteyer et al., 2009; Pikula et al., 2017; Reeder et al., 2012).

65 Following skin surface colonization, $P$. destructans invades the skin and forms

66 cupping erosions diagnostic of WNS (Pikula et al., 2017). A cupping erosion is a cup-

67 shaped skin lesion densely packed with fungal hyphae (Meteyer et al., 2009) and the

68 fungus in the cupping erosions produces secondary metabolites that further damage 
69 skin of the host (Flieger et al., 2016). Early methodology designed to assess disease

70 severity was lethal, as it required excision and rolling an interdigital segment of the

71 wing in preparation for serial cross-sections used in stained microscopy slides.

72 Number and distribution of the skin lesions were then used to score the severity of the

73 disease (Reeder et al., 2012). More recent methodology utilizes punch biopsy

74 sampling guided by characteristic fluorescence of skin lesions in ultraviolet (UV)

75 light, enabling the bat to survive the examination (Pikula et al., 2017).

76 Progress from lethal to destructive sampling facilitated examination of bats with

77 higher conservation status for WNS. To further reduce handling and disturbance of

78 bats for research purposes, herein we estimated the fungal load that invaded host

79 tissues in WNS skin lesions. Our approach combines previously published data on

80 fungal load estimated as the amount of $P$. destructans DNA on the wing punch where

81 the wing was without a WNS lesion and fungal load in a wing punch where UV

82 transillumination revealed characteristic fluorescence indicative of WNS lesions. The

83 relationship between the fungal loads measured from the paired biopsies can be used

84 to estimate how much fungal DNA there is in the bat wing tissue, because $P$.

85 destructans fluoresces under UV light after it had formed skin lesions. As the invasive

86 infection progresses, multiple lesions develop and the fungal load in the skin tissue

87 exceeds the fungal load on the wing surface (Martínková et al., 2018). Sampling in

88 the field cannot distinguish between a single microscopic lesion and multiple

89 confluent lesions as both cases manifest as a fluorescing dot when handling a live bat

90 in a cave (Fig. 1). In the laboratory, quantifying pathogen DNA requires enzymatic

91 tissue digestion, meaning that estimating infection intensity with DNA-based methods

92 as well as disease severity with histological microscopy is not possible from the same

93 sample. We therefore simulate possible histopathologic findings in the wing punch 
94 digested for pathogen DNA quantification to infer how much P. destructans there is

95 in a single WNS skin lesion. We further calculate how the invasive fungal DNA

96 quantity relates to the biology of the infection, and we estimate the number of nuclei

97 in the invading fungal hyphae. We use these results to compare the invasive infection

98 in the Palearctic and Nearctic bats.

99

\section{2. Materials and Methods}

101 We used previously published data on fungal load present in a wing punch biopsy

102 (Fig. 1a in Flieger et al., 2016). The data comes from paired wing punch samples from

103 the same bat, where one punch was taken over a membrane segment with an orange

104 yellow fluorescence spot indicating a lesion (Turner et al., 2014) and the other punch

105 from an area where no UV florescent lesions were apparent (Fig. 1). In total, 41

106 Myotis myotis bats with paired wing biopsies were sampled and fungal load as a

107 measure of fungal DNA was quantified with quantitative polymerase chain reaction

108 (Flieger et al., 2016), i.e. sample size $n=41$.

109 The dependence of the total fungal load on wing membrane that contains a UV

110 fluorescent lesion diagnostic for WNS $P d$ on the fungal load representing surface

111 colonization on an infected wing membrane $\widetilde{P d}$ is linear in logarithmic scale (Flieger

112 et al., 2016). The total fungal load in a biopsy with a lesion $P d$ is compounded from

113 the surface skin colonization $\widetilde{P d}$ and the invasive fungal growth, where the invasive

114 fungal growth is proportional to the number of skin lesions present in the sample.

115 The dependence of $\log _{10}(P d)$ on $\log _{10}(\widetilde{P d})$ can be used to quantify fungal load in a

116 WNS lesion. Once translated to the quadrant IV of the Cartesian system, the intercept

117 of the linear model $\alpha_{0}$ represents the fungal load in the single lesion $\widetilde{P d}^{1}$. The

118 modified relationship is given as 


$$
\log _{10}(P d)=\alpha_{0}+\alpha_{1}\left(\log _{10}(\widetilde{P d})+c\right), \quad \text { Eq. } 1
$$

119 where the constant for translating the data values $c=-\min \log _{10}(\widetilde{P d})$. The

120 unknown model parameters $\alpha_{0}$ and $\alpha_{1}$ can be estimated using a least square method

121 from the set of observations.

122 Following Eq. 1, the fungal load in a single WNS lesion is $\widetilde{P d}^{1}=10^{\alpha_{0}}$ for $\alpha_{1} \approx 1$.

123 Knowing the fungal load in a single WNS lesion and having enumerated the number

124 of WNS lesions on a wing membrane from their UV fluorescence $n_{U V}$, we can

125 calculate the tissue invasive fungal load $\widetilde{P d}$ as

$$
\widetilde{P d}=n_{U V} \cdot \widetilde{P d}^{1}
$$

126 In the empirical study (Flieger et al., 2016), the slope of the regression $\alpha_{1}=0.294$,

127 meaning that difference in fungal load between a biopsy negative and positive for UV

128 fluorescence is due to additional factors than simple presence of a single WNS lesion

129 in one biopsy. To investigate why there is a departure from 1, we deparsed the

130 covariates confounding total fungal load of the $i$-th single wing membrane biopsy

131 containing a UV fluorescent lesion $P d_{i}$. First, the surface of a WNS lesion influences

132 the area, where the fungus colonizes bat wing surface. Second, a UV fluorescent spot

133 observed without magnification in the field might represent a more complex

134 histopathology detectable with microscopy.

136 2.1. Influence of wing surface area of a WNS lesion

137 The first confounding aspect we considered influences intercept $\alpha_{0}$. The surface

138 colonization affects a smaller area in a biopsy with a lesion as the wing surface area of

139 the lesion cannot be considered to contain surface colonization by $P$. destructans. The

140 total fungal load in the $i$-th wing biopsy is 


$$
P d_{i}=\widetilde{P d}_{i}^{*}+\widetilde{P d}_{i}, \quad \text { Eq. } 3
$$

141 where $\widetilde{P d}_{i}$ represents fungal load in the UV fluorescent lesion for the $i$-th biopsy, and

142 for this biopsy, $\widetilde{P d_{i}^{*}}$ represents a surface colonization satisfying the condition of a

143 sampled area $A$. It holds that

$$
A\left(\widetilde{P d}_{i}^{*}\right)=A\left(\widetilde{P d}_{i}\right) \not \subset A\left(P d_{i}\right), \quad \forall i \in I, \quad \text { Eq. } 4
$$

144 where $I$ is a set of sample indices and $I=\{1, \ldots, n\}$.

145 In the sampling regime used to derive the original model (Flieger et al., 2016), the

146 wing membranes were biopsied with standard $4 \mathrm{~mm}$ punch needles. The fungal DNA

147 was thus quantified from the whole wing biopsy with radius $R$, meaning that the

148 surface colonization covers both sides of the biopsy. At least one cupping erosion

149 with radius $r$ was present in the biopsy, and $46 \%$ of $M$. myotis biopsies contained

150 multiple cupping erosions (Pikula et al., 2017), i.e. the area covered by a surface

151 colonization in the $i$-th biopsy is given as

$$
A\left(\widetilde{P d_{i}^{*}}\right)=2 \pi R_{i}^{2}-\pi r_{i}^{2}-0.46 \pi r_{i}^{2} . \quad \text { Eq. } 5
$$

152 In the empirical studies, the diameter of the $i$-th wing punch biopsy was $2 R_{i}=4 \mathrm{~mm}$

153 (Flieger et al., 2016) and mean cupping erosion was $2 r_{i}=86 \mu \mathrm{m}$ (Zukal et al., 2016)

154 for $\forall i \in I$. Solving Eq. 5 numerically shows that $A\left(P d_{i}\right)-A\left(\widetilde{P d}_{i}\right)>0.99 A\left(P d_{i}\right)$

155 for $\forall i \in I$. The effect of presence of the cupping erosion area on the estimation of the

156 fungal load in a WNS lesion is thus negligible.

\section{$158 \quad 2.2$ Influence of unobserved histopathology}

159 The second aspect affects the regression slope $\alpha_{1}$. The UV fluorescent lesion

160 recognized without magnification during field sampling could represent a confluent

161 series of cupping erosions (Fig. 1), increasing the relative invasive growth. The fungal

162 load of wing biopsies with UV fluorescence should be revised according to 


$$
P d^{\prime}=P d-\lambda u, \quad \text { Eq. } 6
$$

163 where $P d^{\prime}$ is the revised fungal load in a wing biopsy with UV fluorescence, $P d$ is the

164 total fungal load estimated from the biopsy, $u$ is number of additional cupping

165 erosions, and $\lambda$ is a theoretical fungal load in the UV fluorescent lesion where

$166 \lambda \in\left[0.1,10^{\alpha_{0}}\right]$ for $\alpha_{0}=-1.01527$. The value for $\alpha_{0}$ was inferred from the least

167 squares regression as per Eq. 1 from the original data, where each biopsy with a UV

168 fluorescence was assumed to contain a single WNS lesion. Note that the published

$169 \alpha_{0}=0.015$ (Flieger et al., 2016) cannot be used directly, because the original data

170 were not translated following their logarithmic transformation. The published data

171 values are located in the quadrant III of the Cartesian system, where the intercept of

172 the regression does not correspond to the fungal load in a single WNS lesion.

173 In our experience, up to four additional cupping erosions can be associated with one

174 biopsy, i.e. $u \in\{0,1,2,3,4\}$ (Fig. 1). Each biopsy in a set of $n$ biopsies is associated

175 with one $u$ forming a set of alternative histopathologic findings $U$ of $n$ elements $u$.

176 According to the results (Pikula et al., 2017), probability of multiple UV fluorescent

177 lesions in the set of biopsies is $p(u \mid u>0)=0.46$, i.e. $p(u \mid u=0)=0.54$. The

178 probability of observing $n_{U V+}$ additional cupping erosions in biopsies with multiple

$179 \mathrm{UV}$ fluorescent lesions is given as $p\left(n_{U V+}\right)=0.5-0.1 n_{U V+}$, where $n_{U V+} \in N_{U V+}$,

180 and $N_{U V+}=\{1,2,3,4\}$. It means that $p(u \mid u>0)=0.46(0.5-0.1 u)$ for $u \in N_{U V+}$.

181 Given the uncertainty in theoretical fungal load in a UV fluorescent lesion $\lambda$, there is

182 an infinite number of possible revised fungal loads (Eq. 6) for the available empirical

183 data. We approached the problem using simulations to estimate the effect of multiple

$184 \mathrm{UV}$ fluorescent lesions in the biopsy on the regression slope $\alpha_{1}$ for sampled

185 combinations of $\lambda$ and $u$. For that purpose, we created a vector $\lambda$ with $n_{\lambda}$ evenly

186 distributed $\lambda$ where $\lambda \in\left[0.1,10^{\alpha_{0}}\right]$, and we included the estimations of the total 
187 fungal loads $P d$ for all $n$ biopsies with a UV fluorescent lesion into a vector $\boldsymbol{P d}$.

188 Further, we generated randomly $n_{u}$ permutations of the set $U$ for each $\lambda \in \lambda$. The $j$-th

189 permutation of $U$ for the $k$-th $\lambda$ is given as $\boldsymbol{u}_{j, k}=\rho_{j, k}(U)$ where $\rho_{j, k}$ is the $j$-th

190 mapping for the $k$-th $\lambda$. In total, $n_{u} \cdot n_{\lambda}$ random vectors $\boldsymbol{u}$ are generated, where the

191 length of the vector $\boldsymbol{u}_{j, k}$ is $n$ for $\forall j \in\left\{1, \ldots, n_{u}\right\}$ and $\forall k \in\left\{1, \ldots, n_{\lambda}\right\}$. For the $k$-th $\lambda$ and

192 the $j$-th permutation, the revised fungal loads in wing biopsies are given as

$$
\boldsymbol{P d}_{j, k}^{\prime}=\boldsymbol{P d}-\lambda_{k} \boldsymbol{u}_{j, k}
$$

193 We used the obtained revised fungal loads $P d^{\prime}$ (Eq. 7) instead of the measured total

194 fungal loads $P d$ while estimating the coefficients $\alpha_{0}$ and $\alpha_{1}$ of the model Eq. 1

195 (Fig. 2). Using the least squares estimator, $n_{u} \cdot n_{\lambda}$ coefficient estimations, $\hat{\alpha}_{0}$ and $\hat{\alpha}_{1}$,

196 were obtained for the $n_{u} \cdot n_{\lambda}$ revised datasets. For each $\lambda \in \lambda$, we searched for

197 coefficient estimations $\hat{\alpha}_{0}$ and $\hat{\alpha}_{1}$ that best match the expected dependence, i.e. we

198 searched for such setting where $\hat{\alpha}_{1} \approx 1$. Considering this, we proposed an objective

199 function

$$
J=\left|1-\hat{\alpha}_{1}^{j, k}\right|, \quad \text { Eq. } 8
$$

200 where $\hat{\alpha}_{1}^{j, k}$ is the estimation of the coefficient $\alpha_{1}$ for $\lambda_{k}$ and the $j$-th permutation $\boldsymbol{u}_{j, k}$.

201 For $\lambda_{k} \in \lambda$, the best setting is given as

$$
\hat{\alpha}_{0}^{k}, \hat{\alpha}_{1}^{k}=\underset{j=1, \ldots, n_{u}}{\arg \min }\left|1-\hat{\alpha}_{1}^{j, k}\right|, \quad \text { Eq. } 9
$$

202 where $\hat{\alpha}_{0}^{k}$ and $\hat{\alpha}_{1}^{k}$ are the best estimates of the coefficients $\alpha_{0}$ and $\alpha_{1}$ for $\lambda_{k}$. Note that

$203 n_{u}$ coefficient estimations were obtained for each $\lambda \in \lambda$, and we calculated fungal

204 load in a WNS lesion as $\widetilde{P d}_{k}=10^{\widehat{\alpha}_{0}^{k}}$.

205 The study assumes that the first biopsy from the pair represents a wing segment

206 without a WNS lesion, in which $P$. destructans colonized wing surface, and the

207 second biopsy from the pair contains a wing segment with a WNS lesion, where the 
208 fungus invaded the tissue. Theoretically, the difference in fungal loads between the

209 two biopsies should be greater than or equal to the fungal load in the WNS lesion.

210 Sampled data that do not conform to the condition are likely influenced by sampling

211 or laboratory artifacts or by as yet unrecognized natural phenomena. To test for the

212 effect of possible unknown bias, we ran a set of simulations on the original and the

213 reduced data. In the case of the reduced data, we used $\forall k \in\left\{1, \ldots, n_{\lambda}\right\}$ those paired

214 biopsies where $P d-\widetilde{P d}>\lambda_{k}$.

215 The equation Eq. 7 allows negative values of revised fungal load in a wing biopsy

216 with a UV fluorescent lesion for some combinations of $\lambda_{k}$ and $\boldsymbol{u}_{j, k}$, which is

217 biologically not feasible. In the absence of $P$. destructans, fungal load must be equal

218 to zero. We considered simulations based on combinations of $\lambda_{k}$ and $\boldsymbol{u}_{j, k}$ leading to

219 any $P d^{\prime}<0$ as failed. Indexes $k$ for which any element of $\boldsymbol{P} \boldsymbol{d}_{j, k}^{\prime}<0, \forall \boldsymbol{u}_{j, k}$ formed a

220 set of failed simulations $D$, which is a subset of all simulations. Fungal load in a

221 single UV fluorescent lesion was estimated from the sampled distribution as

$$
\widetilde{P d}^{1}=P\left(\widetilde{P d}_{\forall k \notin D}\right)
$$

222 The simulations were run in $\mathrm{R}$ (R Core Team, 2018) with custom scripts,

223 implementing equations Eq. 1, Eq. 7, Eq. 8, Eq. 9 and Eq. 10. Data visualization used

224 package RColorBrewer (Neuwirth, 2014).

225 We then used the estimated $\widetilde{P d}^{1}$ to approximate the number of pathogen nuclei in a

226 skin lesion $N$. Given the genome size of $P$. destructans $G=30.685 \times 10^{6}$ bp

227 (GCA_000184105.1) and the conversion constant between genome mass and its size

$228 q=978 \times 10^{6}$ (Doležel et al., 2003), the number of $P$. destructans nuclei in a single

229 WNS skin lesion is

$$
N=\frac{\widetilde{P d}^{1} \cdot q}{G}
$$

Eq. 11 
230 We applied the results of our simulations to previously published data (Pikula et al.,

231 2017) to infer the invasive fungal load in the Nearctic and Palearctic bats using

232 equations Eq. 2 and Eq. 11.

\section{Results}

235 We ran 1 million simulations of unobserved histopathology during estimation of

236 fungal load in a WNS lesion. For $n_{\lambda}=1000$, we permuted designation of samples

237 with multiple UV fluorescent lesions $n_{u}=1000$ times for each $\lambda_{k}$. When the

238 theoretical fungal load in a UV lesion $\lambda_{k} \in[0.075,0.097] \mathrm{ng}, 127$ simulations failed

239 to find any feasible combination of samples with multiple cupping erosions despite

240 attempting one thousand permutations for each $\lambda_{k}$ (Fig. 3), indicating that the range

241 might represent an upper limit of fungal load in one WNS lesion in M. myotis. Results

242 from 873 successful simulations show that simulating unobserved histopathology

243 improves the objective function value $J \in[0.45,0.80]$ compared to the result from the

244 original data, where $J=0.79$ (Flieger et al. 2016). The probability density with

245 Gaussian kernel of the simulated fungal load in a lesion has the mean equal to 0.0489

$246 \mathrm{ng}$ and standard deviation equal to $0.01134 \mathrm{ng}$ (Fig. 3). This means that one WNS

247 skin lesion contains $\widetilde{P d^{1}}=48.9 \pm 11.34$ pg (mean \pm SD) of P. destructans DNA.

248 The simulated fungal load then translates to $1559 \pm 362$ pathogen nuclei in a WNS

249 lesion.

250 When the data were subset in each simulation to those where $P d-\widetilde{P d}>\lambda_{k}$, the

251 objective function values further decreased to $J \in[0.28,0.58]$ and $\widetilde{P d}^{1}=34.97 \pm$

$2527.42 \mathrm{pg}$ of $P$. destructans DNA. In both simulation modes, using all data and using

253 subsets of data, the theoretical $\lambda$ with the lowest objective function values were about

25450.1 to $68.6 \mathrm{pg}$ and the respective estimated $\widetilde{P d^{1}} \in[11.0,17.1] \mathrm{pg}$. 
255 Using the data on disease severity published by Pikula et al. (2017), the Palearctic

256 bats had $n_{U V}=0.78 \pm 1.44 \mathrm{WNS}$ lesions per $\mathrm{cm}^{2}$ of wing membrane area $(n=$

257 173), which translates to $\widetilde{P d}=38.14 \pm 70.42$ pg of $P$. destructans DNA or hyphae

258 with $1216 \pm 2244$ nuclei that invaded the unit area of host tissues. In Nearctic bats

259 with $n_{U V}=34.73 \pm 26.35 \mathrm{UV}$ fluorescent lesions per $\mathrm{cm}^{2}$ of wing $(n=11)$, the

260 invasive fungal growth contains $\widetilde{P d}=1698.30 \pm 1288.52 \mathrm{pg}$ of $P$. destructans

261 DNA, meaning fungal hyphae with $54129 \pm 41068$ nuclei per $\mathrm{cm}^{2}$ of wing area.

\section{Discussion}

264 The utility in modelling pathogen load from data originating from non-lethal sampling

265 provides unquestionable advantages and insight into disease dynamics. Non-lethal

266 sampling promotes more effective sampling that enables to track distribution,

267 prevalence, spread, infection intensity and disease severity on population level. Our

268 approach simulated unobserved histological severity in a biopsy sample that was used

269 to isolate DNA from the bat as well as the total pathogen biomass in the biopsy.

270 Having established a density distribution of likely fungal loads in one WNS lesion,

271 the researchers can now use the information to infer total fungal biomass that invaded

272 the skin of the hibernating bat. We found that one WNS skin lesion contains about 50

273 pg of $P$. destructans DNA and thus about 1560 genomic copies in the fungal

274 multinucleic hyphae and spores in the lesion. Translating the value into context of

275 published data on the number of UV fluorescent spots that are indicative of the WNS

276 lesions (Pikula et al., 2017), we found that in some Nearctic bats, $1 \mathrm{~cm}^{2}$ of their wing

277 membrane might contain more than $1 \mathrm{ng}$ of pathogen DNA or 54000 pathogen nuclei.

278 The limitation of the present study lies in the fact that values of the objective function

279 Eq. 8 did not approach 0 (Fig. 3a). The objective function minimized difference 
280 between the slope of regression from adjusted data with simulated histopathologic

281 findings and the ideal slope equal to 1 , when the regression intercept would signify

282 the fungal load in a single WNS lesion (i.e. Eq. 9). The lack of convergence towards

283 the optimum may be due to complexity of WNS pathology in the biopsy we did not

284 consider. The WNS lesions have variable size (Zukal et al., 2016) and some animals

285 develop full thickness invasion where the fungus replaces host tissues across the

286 cross-section of the wing membrane (Pikula et al., 2017). Additional noise in the data

287 is likely introduced with precision of the biopsy punch. Trained personnel stretch a

288 bat wing on a clean, firm surface transilluminated with UV light and circles the target

289 area with a punch needle. Although the punch needles have constant diameter, the

290 sampled wing may differ depending on animal movement, needle slippage or local

291 stretch of the wing membrane. The apparent solution suggests careful sampling where

292 the paired biopsies would be taken from wing area equidistant from joints and bones

293 and the punch site would be chosen with help from magnification to pinpoint single

294 UV fluorescent spots of similar size. At this moment, such data is not available, and

295 our simulation provides the best data-driven approximation of the invasive fungal

296 load during a WNS infection.

297 We addressed the potential problems in sampling by reducing the dataset to only

298 those observations where the fungal load on a biopsy without a fluorescing WNS

299 lesion was less than fungal load on the paired biopsy by at least the margin of the

300 theoretical fungal load in a single WNS lesion. The change resulted in lower objective

301 function values, but in no simulation was $J \approx 0$. The uncertainty in the estimate of the

302 fungal load in a single WNS lesion remains influenced by the issues mentioned above.

303 Despite the acknowledged bias, we consider our results useful in infectious disease

304 research of protected bat species, in which using non-destructive methods is 
305 warranted. Photography of a bat wing transilluminated with UV light enables

306 enumeration of the WNS lesions in the laboratory and together with estimation of

307 fungal load on the wing surface using a swab sample, these data can be used to

308 calculate infection invasiveness (Martínková et al. 2018). For practical utility in

309 evaluating invasive fungal growth in an infected bat (Eq. 2), we recommend using the

310 fungal load of $49 \mathrm{pg}$ of $P$. destructans DNA in a single WNS lesion. The higher

311 estimate will better incorporate the unconsidered histopathology and also account for

312 presence of confluent WNS lesions that cannot be distinguished on the photograph of

313 the transilluminated bat wing. Using the value of $49 \mathrm{pg}$ of fungal DNA will thus likely

314 better reflect the biological reality of the infection.

315 Our results provide a valuable tool in assessing invasive infection in endangered

316 hibernating bats on organismal level. Prior to the current study, the disease severity

317 was inferred from focal histopathology in a wing biopsy. Now, the researchers can

318 calculate disease severity as a function of infection invasiveness using non-destructive

319 UV light transillumination (Turner et al., 2014) in conjunction with our results about

320 the fungal load in a WNS lesion.

\section{Acknowledgement}

323 The study was supported by the Czech Science Foundation (Grant No. 17-20286S).

324 The funding agency had no involvement in study design; in the collection, analysis

325 and interpretation of data; in the writing of the manuscript; and in the decision to

326 submit the article for publication. The authors thank Nancy R. Irwin and Jan Zukal for

327 discussions.

329 Declarations of interest: none. 
331 Contributors: NM and JP conceptualized the study, NM designed and implemented

332 the simulation and collated the data, PŠ formalized the mathematical apparatus, NM

333 and PŠ wrote the manuscript to which all authors contributed.

\section{References}

Blehert, D. S., Hicks, A. C., Behr, M., Meteyer, C. U., Berlowski-Zier, B. M., Buckles, E. L., 2009. Bat white-nose syndrome: An emerging fungal pathogen? Science 323, 227, doi:10.1126/science.1163874.

Cunnington, A. J., 2015. The importance of pathogen load. PLoS Pathog 11, e1004563, doi:10.1371/ journal.ppat.1004563.

Doležel, J., Bartoš, J., Voglmayr, H., Greilhuber, J., 2003. Nuclear DNA content and genome size of trout and human. Cytometry A 51, 127-128, doi:10.1002/cyto.a.10013.

Flieger, M., Bandouchova, H., Cerny, J., Chudíčková, M., Kolarik, M., Kovacova, V., Martínková, N., Novák, P., Sebesta, O., Stodůlková, E., Pikula, J., 2016. Vitamin B2 as a virulence factor in Pseudogymnoascus destructans skin infection. Scientific Reports 6, 33200, doi:10.1038/srep33200.

Franz, A., Adams, O., Willems, R., Bonzel, L., Neuhausen, N., Schweizer-Krantz, S., Ruggeberg, J. U., Willers, R., Henrich, B., Schroten, H., Tenenbaum, T., 2010. Correlation of viral load of respiratory pathogens and co-infections with disease severity in children hospitalized for lower respiratory tract infection. $\mathbf{J}$ Clin Virol 48, 239-45, doi:10.1016/j.jcv.2010.05.007.

Lorch, J. M., Meteyer, C. U., Behr, M. J., Boyles, J. G., Cryan, P. M., Hicks, A. C., 2011. Experimental infection of bats with Geomyces destructans causes whitenose syndrome. Nature 480, 376-378.

Martínková, N., Pikula, J., Zukal, J., Kovacova, V., Bandouchova, H., Bartonička, T., Botvinkin, A. D., Brichta, J., Dundarova, H., Kokurewicz, T., Irwin, N. R., Linhart, P., Orlov, O. L., Piacek, V., Škrabánek, P., Tiunov, M. P., Zahradníková, A., Jr., 2018. Hibernation temperature-dependent Pseudogymnoascus destructans infection intensity in Palearctic bats. Virulence, doi:10.1080/21505594.2018.1548685.

Meteyer, C. U., Buckles, E. L., Blehert, D. S., Hicks, A. C., Green, D. E., ShearnBochsler, V., 2009. Histopathologic criteria to confirm white-nose syndrome in bats. J. Vet. Diagn. Invest. 21, 411-414, doi:10.1177/104063870902100401. Neuwirth, E., 2014. RColorBrewer: ColorBrewer Palettes. R package version 1.1-2. Pikula, J., Amelon, S. K., Bandouchova, H., Bartonicka, T., Berkova, H., Brichta, J., Hooper, S., Kokurewicz, T., Kolarik, M., Kollner, B., Kovacova, V., Linhart, P., Piacek, V., Turner, G. G., Zukal, J., Martinkova, N., 2017. White-nose syndrome pathology grading in Nearctic and Palearctic bats. PLoS One 12, e0180435, doi:10.1371/journal.pone.0180435. 
372

373

374

375

376

377

378

379

380

381

382

383

384

385

386

387

388

389

390

391

392

393

394

395

396

397
Reeder, D. M., Frank, C. L., Turner, G. G., Meteyer, C. U., Kurta, A., Britzke, E. R., Vodzak, M. E., Darling, S. R., Stihler, C. W., Hicks, A. C., Jacob, R., Grieneisen, L. E., Brownlee, S. A., Muller, L. K., Blehert, D. S., 2012. Frequent Arousal from Hibernation Linked to Severity of Infection and Mortality in Bats with White-Nose Syndrome. PLoS ONE 7, e38920, doi:10.1371/journal.pone.0038920.g001.

Team, R. C., 2018. R: A language and environment for statistical computing. R Foundation for Statistical Computing, Vienna, Austria.

Turner, G. G., Meteyer, C. U., Barton, H. D., Gumbs, J. F., Reeder, D. M., Overton, B., Bandouchova, H., Bartonička, T., Martínková, N., Pikula, J., Zukal, J., Blehert, D. S., 2014. Nonlethal screening of bat-wing skin with the use of ultraviolet fluorescence to detect lesions indicative of white-nose syndrome. J. Wildl. Dis. 50, 566-573, doi:10.7589/2014-03-058.

van der Poll, T., Opal, S. M., 2008. Host-pathogen interactions in sepsis. Lancet Infect. Dis. 8, 32-43, doi:10.1016/s14733099(07)70265-7.

Wilson, D. P., Law, M. G., Grulich, A. E., Cooper, D. A., Kaldor, J. M., 2008.

Relation between HIV viral load and infectiousness: a model-based analysis. The Lancet 372, 314-320, doi:10.1016/s0140-6736(08)61115-0.

Zukal, J., Bandouchova, H., Brichta, J., Cmokova, A., Jaron, K. S., Kolarik, M., Kovacova, V., Kubátová, A., Nováková, A., Orlov, O., Pikula, J., Presetnik, P., Šuba, J., Zahradníková, A., Jr., Martínková, N., 2016. White-nose syndrome without borders: Pseudogymnoascus destructans infection confirmed in Asia. Scientific Reports 6, 19829, doi:10.1038/srep19829. 
398 Figure captions

399 Fig. 1. Scheme of the relation between the biological and the mathematical model

400 in estimating fungal load in a skin lesion. Bat wing membrane (pink) was biopsied

401 at two places, once at a wing segment without orange-yellow fluorescence under UV

402 light, and once at a wing segment with a single fluorescing dot. Each biopsy was

403 enzymatically digested and total fungal DNA was quantified with a quantitative

404 polymerase chain reaction. The quantity of fungal DNA, the fungal load, in a biopsy

405 without UV fluorescence represents the fungus that grows on the wing surface (purple

406 lines, $\widetilde{P d})$. The fungal load in a biopsy with a UV fluorescent dot represents the

407 fungus growing on the wing surface, as well as the fungus that invaded the bat wing

408 and formed cupping erosions diagnostic of white-nose syndrome (purple lines and

409 ovals, $P d$ ). In the field, a single cupping erosion cannot be distinguished from a series

410 of confluent cupping erosions. The unobserved histopathology must thus be simulated

411 to estimate the fungal load in a single cupping erosion $\left(\widetilde{P d}^{1}\right)$.

413 Fig. 2. Illustrative modification of fungal load in a bat wing membrane biopsy 414 with UV fluorescence to include simulation of the histopathologic findings. Open

415 circles - measured quantification of Pseudogymnoascus destructans DNA in paired

416 biopsies from one bat with the respective regression between fungal load in a wing

417 biopsy without UV fluorescence (and thus without a WNS lesion) and fungal load in a

418 biopsy with UV fluorescence given as a dashed line. Closed circles - adjusted fungal

419 loads, mimicking putative presence of multiple WNS lesions in one biopsy (Eq. 7).

420 The regression line is solid for the adjusted fungal loads. Arrows - direction of

421 change from measured to simulated fungal load per one WNS lesion in the biopsy.

422 Where no arrows are present, the closed circles overlap the measured data in the open 
423 circles, meaning that the simulation did not assign more than one WNS lesion in the

424 given biopsy. $\lambda$ - Theoretical fungal load in one WNS lesion as used in the simulation

$425 \quad(n g)$.

426

427 Fig. 3. Load of Pseudogymnoascus destructans DNA in one UV fluorescent lesion

428 diagnostic for white-nose syndrome in a Myotis myotis bat. (A) Successful

429 simulations of permuted number of additional WNS skin lesions in a wing biopsy.

430 Theoretical starting values of fungal load in a single WNS lesion are indicated by the

431 colour scheme. (B) Density with the Gaussian kernel of the sampled distribution of

432 fungal load in a single WNS lesion in successful (orange) and failed (black)

433 simulations. Simulations were considered failed if all random assignments of

434 additional WNS lesion in a biopsy resulted in at least one adjusted fungal load below

435 zero.

436 


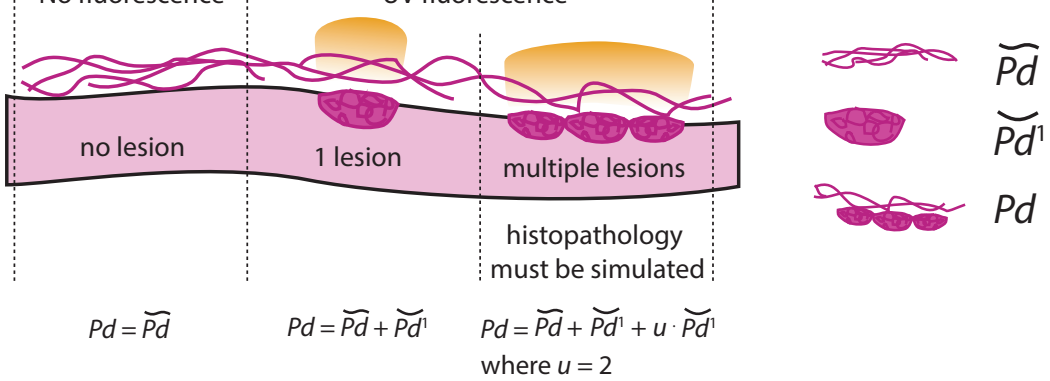


a)

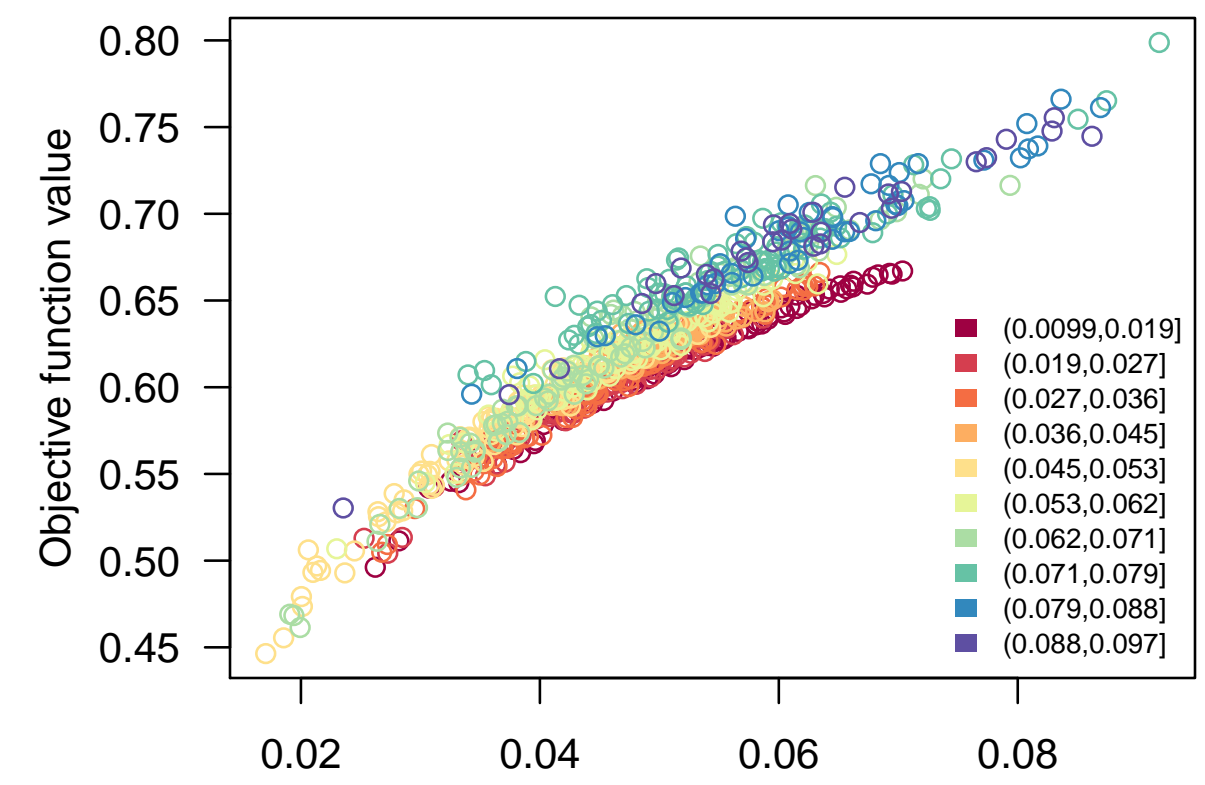

Estimated fungal load in one WNS lesion (ng)

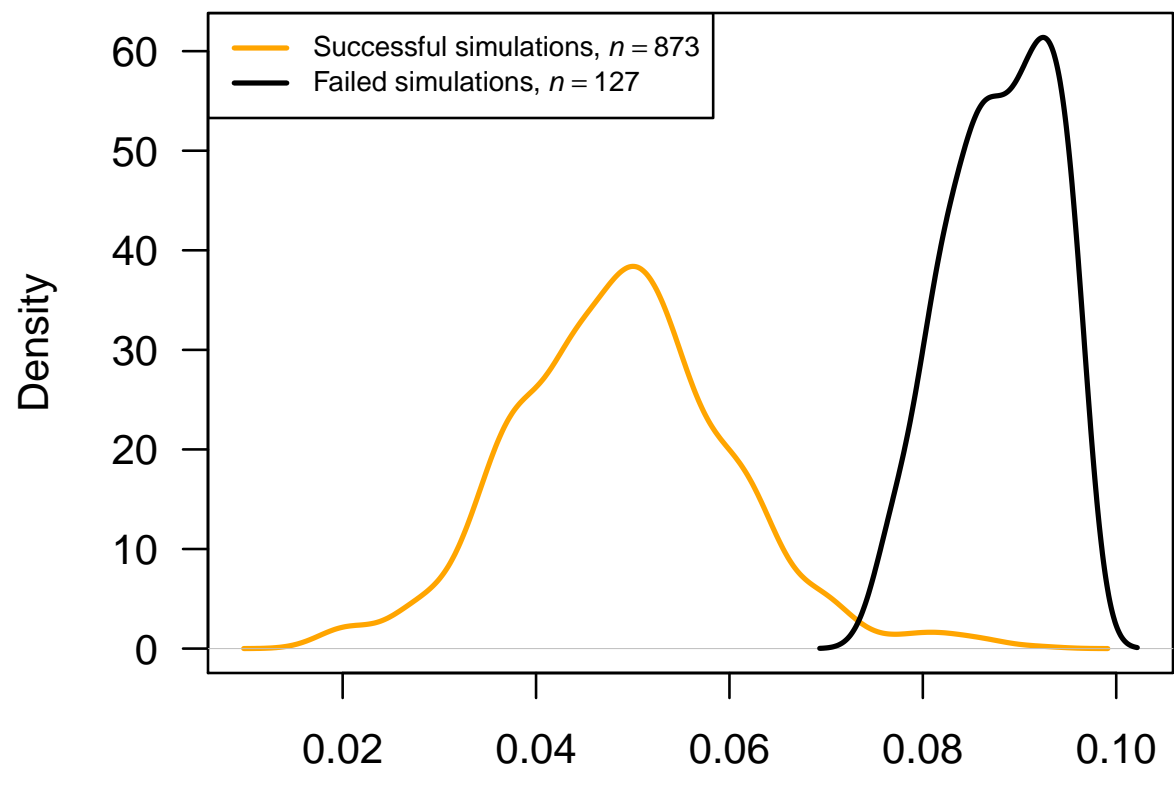

Fungal load in one WNS lesion (ng) 